\title{
Article \\ Alteration of 3D Matrix Stiffness Regulates Viscoelasticity of Human Mesenchymal Stem Cells
}

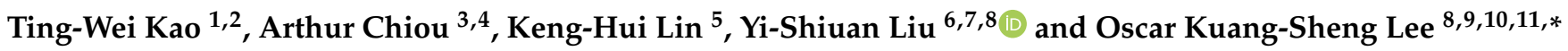 \\ 1 Department of Medical Education, National Taiwan University Hospital, Taipei 100, Taiwan; \\ twkao315@gmail.com \\ 2 Faculty of Medicine, National Yang-Ming University, Taipei 100, Taiwan \\ 3 Institute of Biophotonics, National Yang-Ming University, Taipei 112, Taiwan; aechiou@ym.edu.tw \\ 4 Biophotonics and Molecular Imaging Research Center, National Yang-Ming University, Taipei 112, Taiwan \\ 5 Institute of Physics, Academia Sinica, Taipei 115, Taiwan; khlin@phys.sinica.edu.tw \\ 6 Department of Physiology and Pharmacology, Chang Gung University College of Medicine, \\ Taoyuan 333, Taiwan; lyspub1@gmail.com \\ 7 Department of Plastic and Reconstructive Surgery, Chang Gung Memorial Hospital, Taoyuan 333, Taiwan \\ 8 Institute of Clinical Medicine and Stem Cell Research Center, National Yang-Ming University, \\ Taipei 115, Taiwan \\ 9 Department of Medical Research, Taipei Veterans General Hospital, Taipei 115, Taiwan \\ 10 Department of Orthopedics, School of Medicine, China Medical University, Taichung 404, Taiwan \\ 11 Department of Orthopedics, China Medical University Hospital, Taichung 404, Taiwan \\ * Correspondence: oscarlee9203@gmail.com
}

check for updates

Citation: Kao, T.-W.; Chiou, A.; Lin, K.-H.; Liu, Y.-S.; Lee, O.K.-S. Alteration of 3D Matrix Stiffness Regulates Viscoelasticity of Human Mesenchymal Stem Cells. Int. J. Mol. Sci. 2021, 22, 2441. https://doi.org/ $10.3390 /$ ijms 22052441

Academic Editor: Vladislav Volarevic

Received: 13 January 2021

Accepted: 25 February 2021

Published: 28 February 2021

Publisher's Note: MDPI stays neutral with regard to jurisdictional claims in published maps and institutional affiliations.

Copyright: (c) 2021 by the authors. Licensee MDPI, Basel, Switzerland. This article is an open access article distributed under the terms and conditions of the Creative Commons Attribution (CC BY) license (https:// creativecommons.org/licenses/by/ $4.0 /)$.

\begin{abstract}
Human mesenchymal stem cells (hMSCs) possess potential of bone formation and were proposed as ideal material against osteoporosis. Although interrogation of directing effect on lineage specification by physical cues has been proposed, how mechanical stimulation impacts intracellular viscoelasticity during osteogenesis remained enigmatic. Cyto-friendly 3D matrix was prepared with polyacrylamide and conjugated fibronectin. The hMSCs were injected with fluorescent beads and chemically-induced toward osteogenesis. The mechanical properties were assessed using video particle tracking microrheology. Inverted epifluorescence microscope was exploited to capture the Brownian trajectory of hMSCs. Mean square displacement was calculated and transformed into intracellular viscoelasticity. Two different stiffness of microspheres $(12 \mathrm{kPa}, 1 \mathrm{kPa})$ were established. A total of 45 cells were assessed. hMSCs possessed equivalent mechanical traits initially in the first week, while cells cultured in rigid matrix displayed significant elevation over elastic $\left(\mathrm{G}^{\prime}\right)$ and viscous moduli $\left(\mathrm{G}^{\prime \prime}\right)$ on day $7(p<0.01)$ and $14(p<0.01)$. However, after two weeks, soft niches no longer stiffened hMSCs, whereas the effect by rigid substrates was consistently during the entire differentiation course. Stiffness of matrix impacted the viscoelasticity of hMSCs. Detailed recognition of how microenvironment impacts mechanical properties and differentiation of hMSCs will facilitate the advancement of tissue engineering and regenerative medicine.
\end{abstract}

Keywords: mesenchymal stem cell; osteogenesis; viscoelasticity

\section{Introduction}

Osteoporosis has been a longstanding issue in healthcare, and its prevalence is on constant rising due to aging of society and increased clinical awareness. According to recent epidemiological report, 10 million individuals over the age of 50 in the U.S. endorsed such diagnosis [1], and a substantial portion of this cohort was eventually complicated with a compression fracture. Mechanistically, prolonged phase of bone absorption by osteoclasts and shortened duration of bone formation by osteoblasts brings about pathological bone remodeling [2]. Wolff's law suggested the strength of bone reflects mechanical loading. Specific population under abnormal physical pressure, e.g., bed-ridden individuals and astronauts, are therefore predisposed for osteoporosis. Traditional approach, in addition to a 
rehabilitation program, consisted of medical treatment including as biphosphates, selective estrogen receptor modulators, parathyroid hormone analog, monoclonal antibodies such as denosumab and romosozumab, as well as vitamin D and calcium supplementation [3]. Despite the demonstration of therapeutic efficacy by the mentioned drug noted above, clinically poor responders are still frequently encountered. Development of newer treatments for osteoporosis is needed to better manage osteoporosis.

Bone reconstruction by employing human mesenchymal stem cells (hMSCs) has currently become an alternative. To direct the lineage commitment of hMSCs, literature suggested not only biochemical induction but physical stimuli also ought to be fine-tuned. Studies demonstrated even in the absence of pharmaceutical cues, the rigidity of culture microenvironment impacts cell fate choice [4], as stiff and soft niche respectively stimulates hMSCs toward osteogenesis and adipogenesis. Specifically, high compliance of extracellular matrix influenced MAPK signaling by activating Rho/ROCK and localizing YAP/TAZ into the nucleus, thereby fastening RUNX2 to promote osteogenesis [5,6]. Various parameters of the matrix, including thickness and cross-linking, were proposed to influence lineage specification of indwelling stem cells [7]. Furthremore, dimensionality was proposed to be the crucial parameter for induction of pluripotency and differentiation [8,9]. Hsieh et al. also suggested in conjunction with matrix stiffness, the dimensionality synergistically orchestrated the expression of osteogenic marker genes of hMSCs [10]. Rather than conventional culture method, three dimensional scaffolds more precisely recapitulated the in vivo condition. This biomimetic niche facilitated the advancement of tissue engineering [11], and the optimized spherical boundary condition offered the platform to investigate cell spreading, migration, and morphogenesis.

Additionally, documentation of the mechanical properties during cell fate commitment is the cornerstone to monitor the progression of osteogenesis. Young's modulus, defining correlation between stress and strain, gradually escalates as the bone formation matures. For most circumstances, the intracellular mechanical properties were assessed by the atomic force microscope (AFM) [12]. However, such measure necessitated the contact of probe tip and cell of interest, which inevitably altered the rigidity of cytoskeleton. Since the hMSCs viscoelasticity was in inverse proportion to the total Brownian motion distance of intracellular particles, video particle tracking microrheology (VPTM) methodology and in conjunction with the generalized Einstein-Stokes equation were exploited to indirectly evaluate the mechanical properties [13].

The aim of this study was to interrogate the mechanical impact of culture niche on hMSCs during osteogenic differentiation, and thereby established the protocol for future investigation on mechanotransduction. Depiction of the study design was exhibited in Figure A1.

\section{Results}

\subsection{Scaffold Profiling and Bead Concentration}

3D porous scaffolds with two distinct rigidities were manufactured for hMSCs culture. Stiffness at $1 \mathrm{kPa}$ and $12 \mathrm{kPa}$ were opted to physiologically recapitulate the mechanical properties of the nervous system and mineralized bone. Pore size of the matrices was controlled uniformly at a $100 \mu \mathrm{m}$ diameter for optimal osteogenesis (Figure 1). The initial concentration of fluorescent beads was $6.2 \times 10^{12}$ particle $/ \mathrm{mL}$. Although a $1 / 20$ dilution ratio is widely applied in $2 \mathrm{D}$ culture setting, the $3 \mathrm{D}$ substrate with additional $\mathrm{z}$ axis inevitably result in the projection overlapping the fluorescent beads and thus lead to misrecognition of the random walk for individual particles. To fine-tune the optimal ratio for dilution, three arbitrary values, $1 / 20,1 / 30$, and $1 / 50$, were examined. Those cells subjected to the injection of $1 / 20$ and $1 / 30$ diluted particles were too crowded to analyze individual behavior of the beads, whilst the $1 / 50$ mixing ratio was eligible to both prevent excessive particle-to-particle disturbance and offer sufficient tracking information (Figure 2). Therefore, all trials in the study were based on this dilutive condition. 


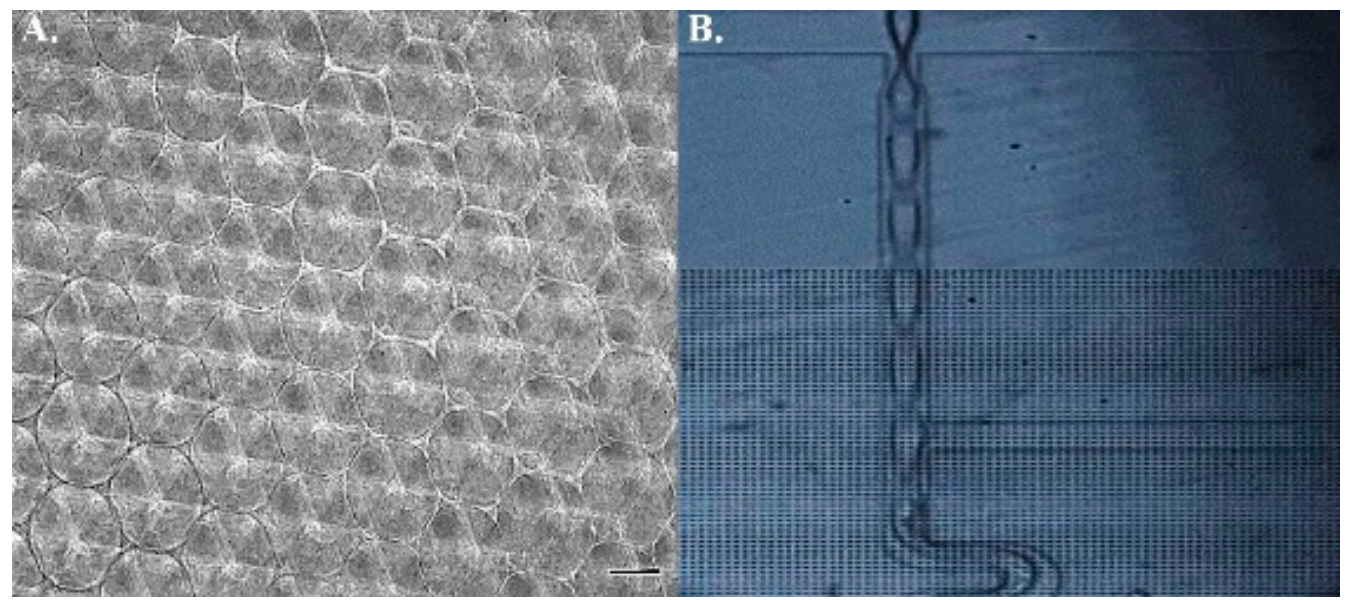

Figure 1. Fabrication of 3D polyacrylamide scaffold. (A) The diameter of each sphere was set at $100 \mu \mathrm{m}$. (B) The microfluidic device was employed to carry nitrogen for polyacrylamide and ammonium persulfate mixing. Scale bar $=100 \mu \mathrm{m}$.

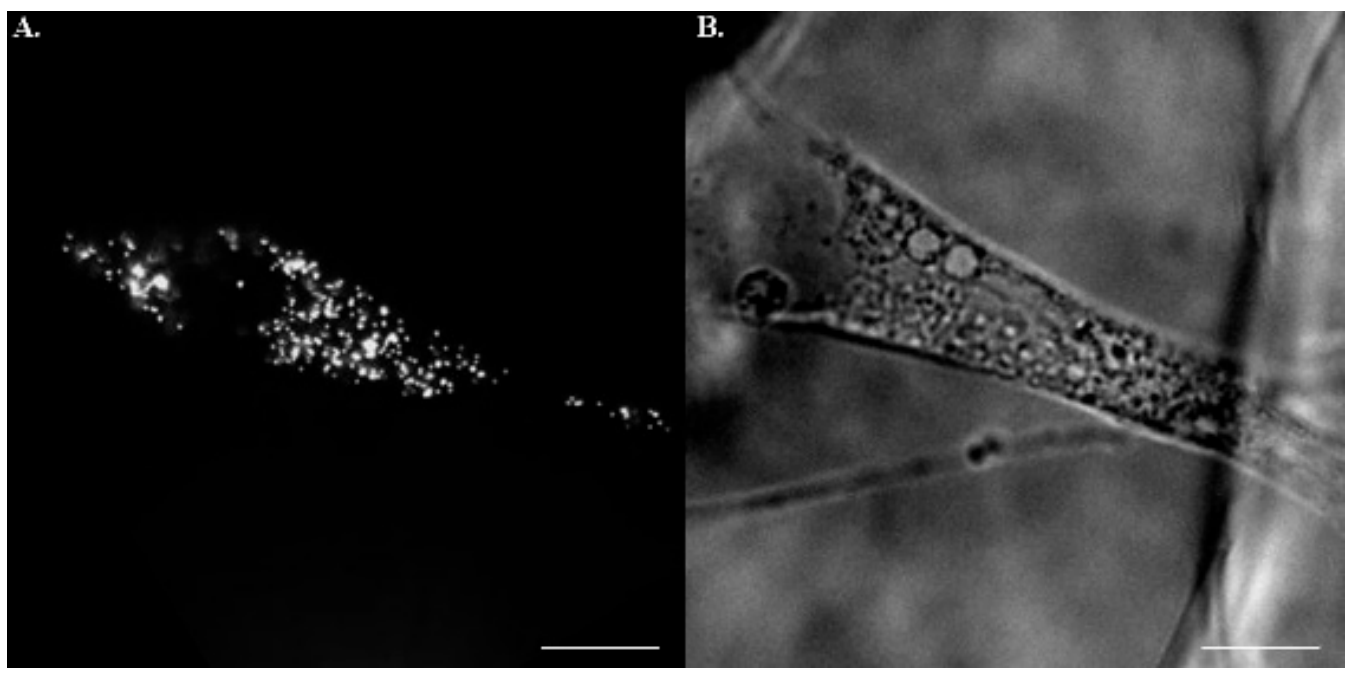

Figure 2. An example of fluorescent beads in hMSCs. Under (A) TxRed and (B) bright field illustration with a charge-coupled camera, the intracellular fluorescent particles were readily identifiable. Scale bar $=20 \mu \mathrm{m}$.

\subsection{Postures of hMSCs in Porous Scaffolds}

Unlike 2D substrates where cultured cells flattened universally, the indwelling hMSCs in 3D scaffolds assumed heterogenous postures, e.g., bridging over (Figure 3A) or vertical lying (Figure 3B). The most frequently encountered morphology was pyramidal-shaped (Figure 3C), of which respectively coplanar but not linear anchoring forces at 3 edges reached static equilibrium as Lami's theorem suggested. An equal number of cells with each morphology were collected for calculation. To study whether or not bone formation would be influenced by fluorescent beads, the expressions of osteogenic markers, Osterix and Runx2, in those cells status post particle injection were quantified and compared with control. Initial elevation of the markers corresponded well with the course of osteogenesis. Additionally, the introduction of fluorescent beads did not bring about a significant difference throughout the differentiation course (Figure 4). 


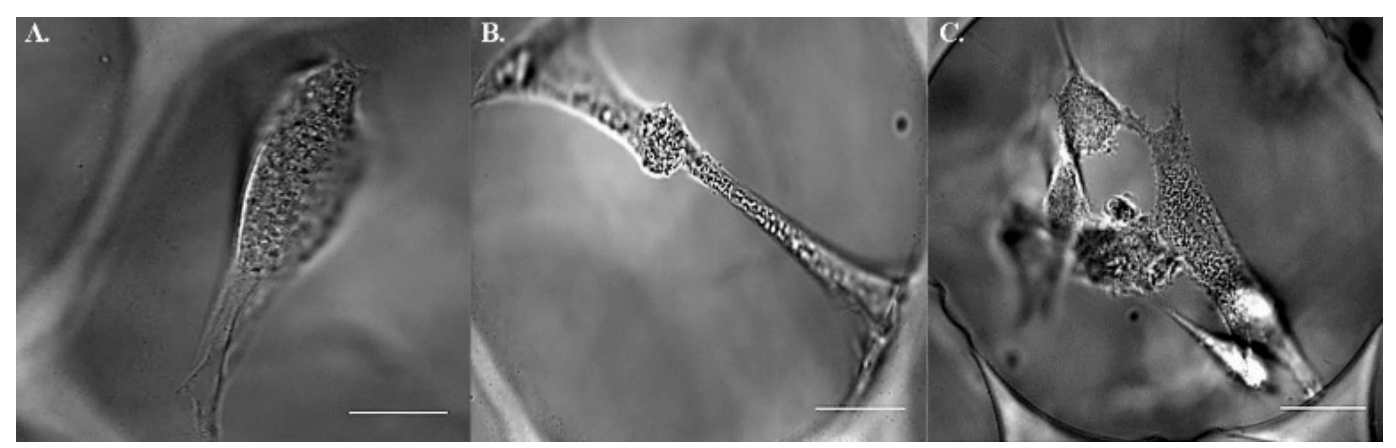

Figure 3. Heterogeneous morphologies of hMSCs indwelling 3D substrates. Bright field of hMSCs (A) vertically lying (B) bridging over (C) pyramidal-shaped. Scale bar $=20 \mu \mathrm{m}$.
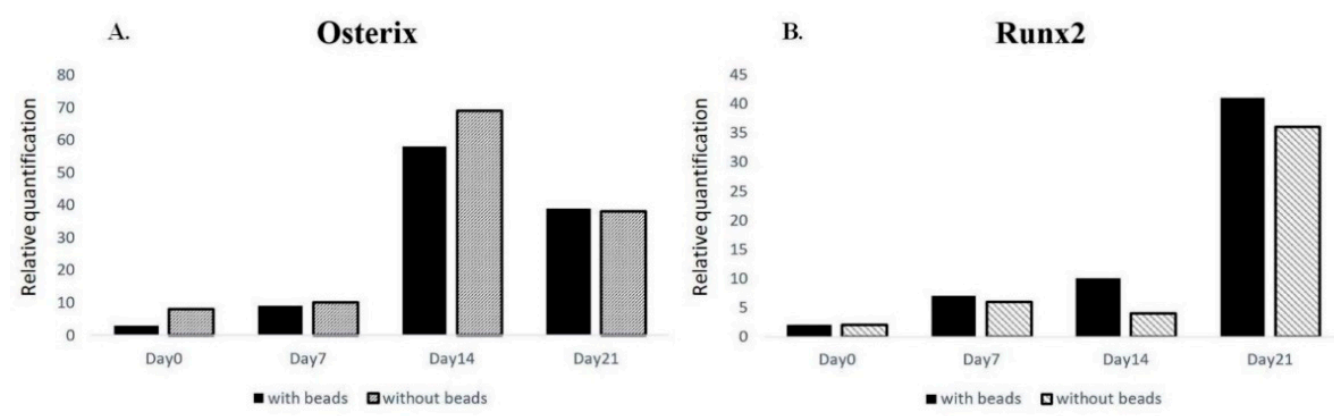

Figure 4. Injected fluorescent beads did not influence hMSCs osteogensis. No significant difference was present in marker gene expressions, (A) Osterix and (B) Runx2, in cells with or without fluorescent particles.

\subsection{Assessment of Intracellular Viscoelasticity by VPTM}

A total of 45 different cells in three independent trials were assessed using confocal microscope. 4 to 10 fluorescent particles per hMSCs were identified for analysis, and every hMSC of interest was snapshotted with both bright field and confocal resolution. Each Brownian motion was traced, and the trajectory was projected to transverse xy-plain for subsequent calculation. The motion was depicted in respective to time and along $\mathrm{x} / \mathrm{y}$ axis individually. Integration thereof yielded mean square displacement, which was applied in the function of time step to generalized Einstein-Stokes equation. (Figure 5). For stable and adequate outcome, the frequency was set as $10 \mathrm{~Hz}$ in the equation to obtain complex shear moduli.

Initially at Day 0 , the mechanical properties of hMSCs in both $1 \mathrm{kPa}$ and $12 \mathrm{kPa}$ group was comparable, and no significant differences in elastic modulus were found by the first week after chemical induction toward osteogenesis. Viscosity of hMSCs in $1 \mathrm{kPa}$ scaffold, however, was up to $168 \mathrm{~Pa}$ and was slightly higher than that in $12 \mathrm{kPa}$ substrate, $135 \mathrm{~Pa}$. Interestingly, hMSCs in the $12 \mathrm{kPa}$ scaffolds exhibited continuously strong increase in both elastic as well as viscous moduli, i.e., $\mathrm{G}^{\prime}$ and $\mathrm{G}^{\prime \prime}, 2$ weeks (192 Pa) and 3 weeks (204 Pa) postinduction. As for the effect posed by soft $1 \mathrm{kPa}$ matrix, the $\mathrm{G}^{\prime}$ and $\mathrm{G}^{\prime \prime}$ fluctuated to (114 $\mathrm{Pa}$, $169 \mathrm{~Pa}$ ) and $(129 \mathrm{~Pa}, 161 \mathrm{~Pa})$, respectively (Figure 6A,B). Therefore, rigid matrix not only biased hMSCs toward bone formation but also elevated the rigidity of the cytoskeleton.

Furthermore, to appreciate the trend of alterations, follow-up measurements were put into comparison with Day 0 initial value in respective groups. Intriguingly, soft $1 \mathrm{kPa}$ niches barely exerted any further effect on viscoelasticity enhancement after Day 7. Rigid $12 \mathrm{kPa}$ matrices, on the contrary, promoted the escalation of both $G^{\prime}$ and $G^{\prime \prime}$ up to $204 \mathrm{~Pa}$ and $222 \mathrm{~Pa}$ in a consistent manner during the entire osteo-specification course (Figure 6C,D), illustrating hMSCs perceived the varied degree of the mechanical impact during bone formation by exhibiting different trends of changes on viscoelasticity in response. 
A.

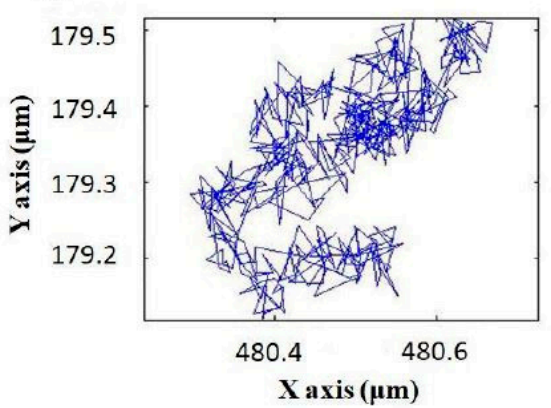

D. Brownian motion alongy axis

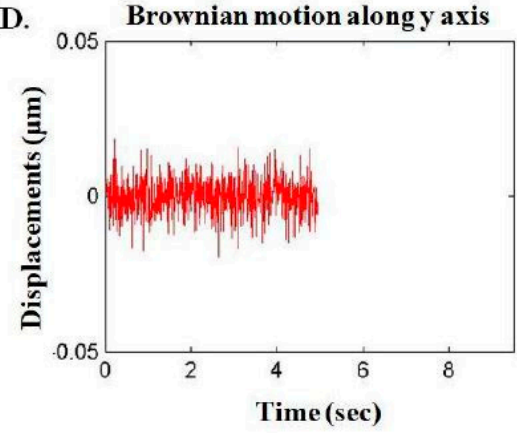

B. Brownian motion along $x$ axis

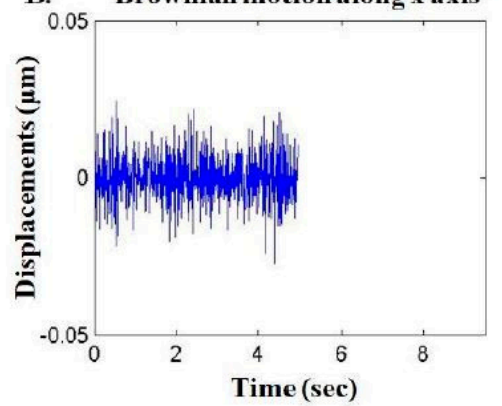

E. Brownian motion alongy axis

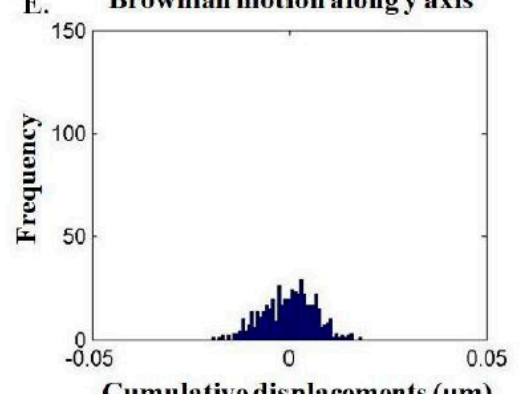

C. Brownian motion along $x$ axis

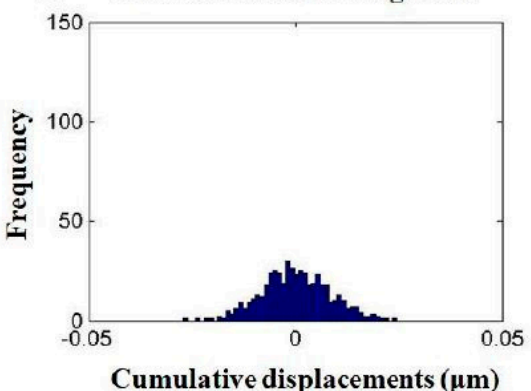

F.

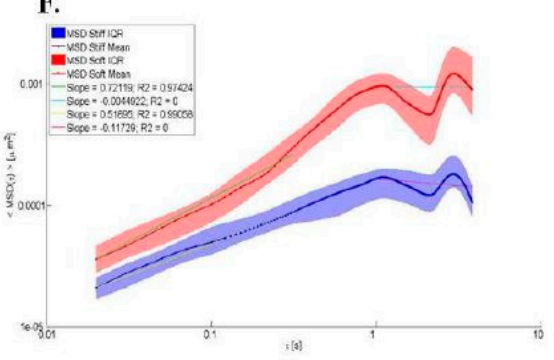

Figure 5. Computation of the hMSCs mechanical properties by passive microrheology. (A) The Brownian motion of beads in hMSC of interest was traced and projected to XY-plain. (B,D) In respect to time, the motion along $x$ and $y$ axis was depicted individually. (C,E) All documented displacements were integrated and expressed with frequency to calculate mean square displacement (MSD). (F) Young's modulus was yielded by applying MSD into generalized Einstein-Stokes equation.

A.

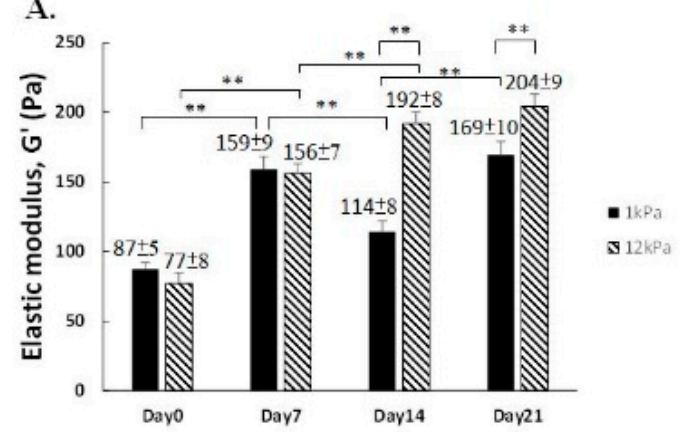

C.

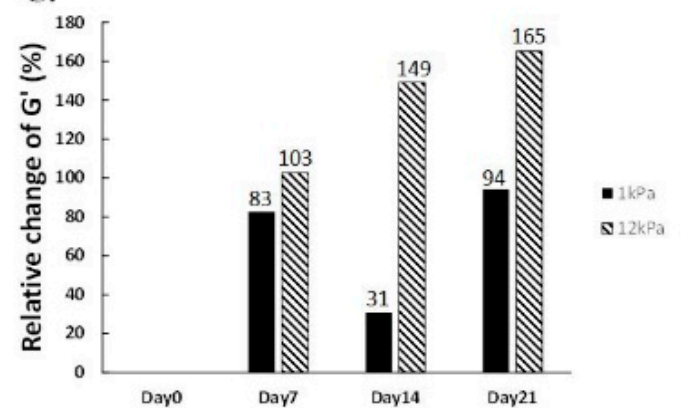

B.

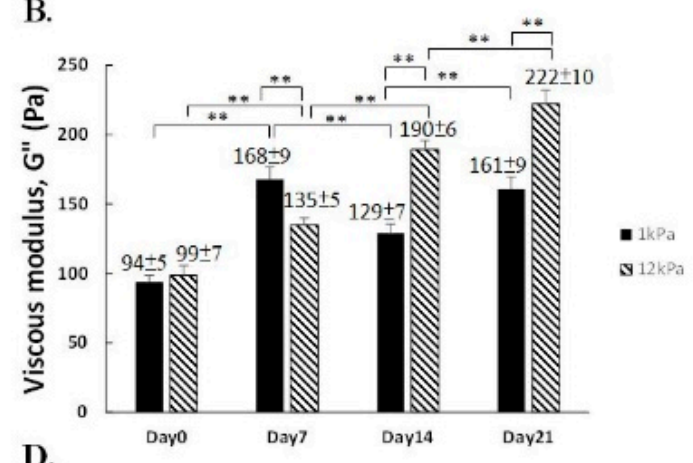

D.

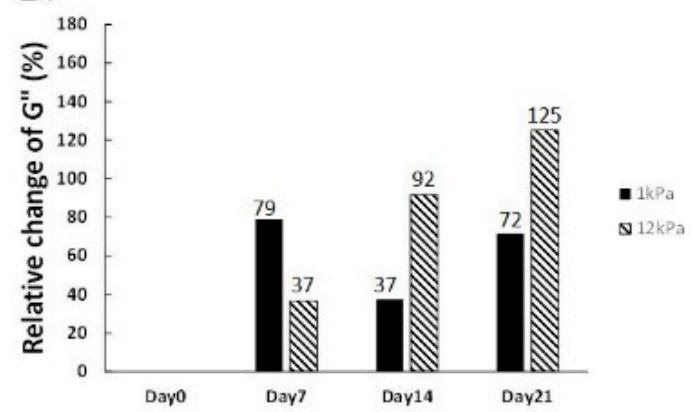

Figure 6. Viscoelasticity of hMSCs during osteogenesis. $(\mathbf{A}, \mathbf{B})$ The elastic $\left(\mathrm{G}^{\prime}\right)$ and viscous modulus $\left(\mathrm{G}^{\prime \prime}\right)$ of hMSCs cultured in stiff and soft substrates. (C,D) Relative changes of Young's modulus in the course of chemically-induced osteogenesis. $\left({ }^{* *} p<0.01\right)$ 


\section{Discussion}

Although it has been well-established that the mechanical properties of residing microenvironment wound bias lineage specification of hMSCs [14], how the architecture of cytoskeleton responded to physical stimuli remained enigmatic. In this study, the culture platform of 3D polyacrylamide scaffold was established, and the alteration of intracellular viscoelasticity was assessed in conjunction with VPTM employment. Osteoblast maturation has been proposed to accompany with the elevation of viscoelasticity, and the substrate stiffness modulated the behavior of osteoblasts [15]. Our study illustrated the viscous and elastic modulus of hMSCs in $12 \mathrm{kPa}$ substrates was on constant increasing in the entire osteogenic course. On the contrary, Young's modulus of those in $1 \mathrm{kPa}$ reached plateau by the second week post chemical induction. $12 \mathrm{kPa}$ was hence the preferable rigidity of $3 \mathrm{D}$ substrates for residence to $1 \mathrm{kPa}$ in terms of both osteolineage commitment and cytoskeleton stiffening. Culturing cells in stiffer 3D scaffolds prior to engraftment ensured the mechanical properties would rise to level of osteoblast and potentially rescue osteoporosis.

To accurately quantify mechanical characteristics, minimization of unintended physical impacts during cell transfer and property measurement was of priority. Aside from cryopreservation so as to halt confounding mechanotranductive effects, traditional AFM was replaced for the assessment of hMSCs mechanical characteristics. To ideally evaluate spatiotemporal rheological properties of hMSCs, the passive microrheology technique, VPTM $[16,17]$, was firstly employed to study Young's modulus during osteogenesis. Soft tissue was especially feasible and already ubiquitous for VPTM measurement. Using diffusing wave spectroscopy, Chenet et al. quantified the viscoelasticity of synovial fluid of arthritis patient [18]. Chen et al. applied VPTM to interrogate viscoelasticity of HeLa cells during cell division [19]. However, each sample required the establishment of individualized protocol and study design. In this study, we pinpointed the dilution ratio of $1 / 50$ for fluorescent particles with phosphate buffered saline, and also assigned the frequency parameter at $10 \mathrm{~Hz}$ for transformation. The protocol could be applied to other hMSCs experiments, while customized modifications might be necessary regarding the study objectives.

As the advancement of tissue engineering and biophysical materials, the concept on 3D matrix for cell indwelling was introduced [20]. Adapted from the experience of fibroblast culture, the negatively curved surface promoted cell adhesion and realized an extra-dimensionally to interrogate cellular behavior. Moreover, the spherically boundary condition was demonstrated to impact hMSCs osteogenesis. Enlightened by previous study, 3D substrates with a diameter at 100 or $150 \mu \mathrm{m}$ were the preferred size for bone formation [21]. In this study, the material for matrix manufacturing was designed universally at $100 \mu \mathrm{m}$ to boost osteogenesis. Previous study pointed out the rigidity of 3D substrate at $11 \sim 30 \mathrm{kPa}$ influenced hMSCs commitment by orchestrating integrin binding and adhesion ligands [22]. Literature also highlighted in 3D setting, the cellular behavior and ligand chemistry responding to substrate stiffness were different [23]. The dissimilarity between topological structures lies in the mechanical cues the niche imposed on the cells within [24]. 2D collagen-coated microenvironment brought about apical-basal polarity, whereas 3D niche was compatible with unconstrained spreading. Interestingly, recent evidence linked the using of 3D matrix as culture condition to the assistance of stem cell reprogramming. The scaffold prepared by $3 \mathrm{D}$ printing was also proposed to improve bone regeneration by bone marrow-derived mesenchymal stem cells [25], and 3D extracellular matrix was illustrated to both offer biophysical stimuli but also preserve tissue [26]. These together underscored the effect of an "extra-dimension" on stem cell physiology.

There are limitations of this study. First, the mechanical background hMSCs experienced before indwelling into the 3D spheres was undetermined. 3D-to-3D transfer was techanically challenging and the protocol was still immature. Egger et al. performed the first isolation of adipocytes from native tissue directly into 3D hydrogel without contacting 2D dish [27]. In our experiments, hMSCs were maintained on hard 2D plastic surface before transferred to 3D culture, thereby resulting in mechanical memory. This confounding 
parameter might interfere cell culture. Manipulation of miR-21 was proposed to erase this effect [28] but no references were available for execution under the circumstance of hMSCs osteogenesis. Second, the mechanical stimulus cultured hMSCs underwent depended on its posture. Topologically different anchorage morphologies detected respective physical cues and thus transduced non-identical signals for proliferation and fate commitment [29]. Even though VPTM was indicated to the single cell level, opted cells of interest were selected manually according to visual selection over cellular morphology and therefore subjective. Development of autonomic calculation and grouping by physical characteristics was mandated for stratified analysis of the viscoelasticity.

As for future prospective, the mechanotransductive pathway for hMSCs is not fully understood. Different types of mechanical loading have been proposed, including shear stress, compression, vibration, etc. Nevertheless, how the cells adapted under various physical signals and the changes in marker gene expression were rarely touched by previous studies. Becquart et al. reported intermittent shear stress and cyclic hydrostatic pressure influenced the production of nitric oxide expression of mechanosensitive genes, thereby harnessing stem cell differentiation [30]. Moreover, the mechanical feedback of cultured hMSCs to the local niche for cell fate manipulation remained enigmatic. Detailed appreciation on the mechanical impacts for hMSCs differentiation is the cornerstone for the development of tissue engineering and materials for regeneration.

\section{Materials and Methods}

\subsection{Fabrication of Polyacrylamide 3D Scaffolds}

Polyacrylamide matrices, unlike gelatin scaffold with otherwise unchangeable rigidity, were manufactured. Fundamental ingredients were acrylamide (Bio-red, Hercules, CA, USA) as monomer and bis-acrylamide (Bio-red, Hercules, CA, USA) as crosslinker. The mixing ratio was $[18.75 \%, 1.13 \%] /[7.00 \%, 0.20 \%]$ for stiff/soft matrices, and former AFM measurements determined the rigidity at $12 \mathrm{kPa}$ and $1 \mathrm{kPa}$, respectively. Other constituents, including gelling initiator ammonium persulfate (Sigma-Aldrich, St. Louis, MO, USA), surfactant Pluronic F-127 (Sigma-Aldrich, St. Louis, MO, USA), and catalyst N, $\mathrm{N}^{\prime} \mathrm{N}^{\prime}, \mathrm{N}^{\prime}$ tetramethylethylenediamine (Sigma-Aldrich, St. Louis, MO, USA), accounted for $1 \%$ of total mixture. Besides, soft-lithographed polydimethylsiloxane-based microfluidic device was exploited to direct the flow and facilitate the mixing of input substances. Polyacrylamide, ammonium persulfate, and nitrogen gas saturated with perfluorohexane (C6F14) were introduced by three individual inlets, and monodisperse foams to fulfill reservoir ( $6 \mathrm{~mm}$ in width, $1 \mathrm{~mm}$ in height) were collected at the conjoint outlet. The semi-solid yield was solidified in $80^{\circ} \mathrm{C}$ oven and degassed in vacuum to patent homogeneous $100 \mu \mathrm{m}$ pores. Scaffolds were eventually treated with $1 \mathrm{mg} / \mathrm{mL}$ sulfosuccinimidyl 6-(4'-azido-2'nitrophenylamino) hexanoic acid (Thermo, Waltham, MA, USA) and activated under 8-min ultraviolet radiation to realize the conjugation of $0.5 \mathrm{mg} / \mathrm{mL}$ fibronectin (Sigma-Aldrich, St. Louis, MO, USA) for cyto-friendly culture condition. Summary of utilized techniques was listed (Table 1).

Table 1. List of the techniques utilized in the study. hMSC: human mesenchymal stem cell.

\begin{tabular}{lll}
\hline Technique & Purpose & Application in this Study \\
\hline $\begin{array}{l}\text { Polydimethylsiloxane-based } \\
\text { microfluidic device }\end{array}$ & $\begin{array}{l}\text { To propel the movement of those ingredients } \\
\text { introduced for scaffold manufacturing }\end{array}$ & $\begin{array}{l}\text { The device was employed to facilitate mixing } \\
\text { of input materials }\end{array}$ \\
\hline Biolistic delivery system & To place particle into cell of interest & $\begin{array}{l}\text { Fluorescent beads were injected } \\
\text { intracellularly to hMSCs }\end{array}$ \\
\hline Charge-coupled camera & $\begin{array}{l}\text { To capture cellular image under } \\
\text { designated resolution }\end{array}$ & $\begin{array}{l}\text { TxRed and bright images of hMSCs } \\
\text { were obtained. }\end{array}$ \\
\hline $\begin{array}{l}\text { Video particle } \\
\text { tracking microrheology }\end{array}$ & $\begin{array}{l}\text { To deduce Young's modulus from Brownian } \\
\text { motion of intracellular particles }\end{array}$ & $\begin{array}{l}\text { The viscoelasticity of hMSCs were yielded by } \\
\text { tracing the random walk of } \\
\text { fluorescent particles }\end{array}$ \\
\hline
\end{tabular}




\subsection{Injection and Tracking of Fluorescent Particles}

To prevent confounding mechanical influences and for execution convenience, the fluorescent beads were injected into hMSCs prior to seeding. The protocol was adapted from preceding reports. Through the propulsion of helium, the biolistic delivery system was applied to introduce $0.2 \mu \mathrm{m}$ red fluorescent carboxylate-modified microspheres. The particle was negatively-charged and possessed maximum emission at the wavelength of $605 \pm 5 \mathrm{~nm}$. These beads randomly walked around the cytoskeleton under otherwise minimal exogenous stimuli except the rigidity of indwelled matrix. The Brownian displacement of fluorescent particles were captured using inverted epifluorescence microscope equipped with charge-coupled device camera and oil immersion objective lens (numerical aperture $=1.45,100 \times$ ).

\section{3. hMSCs Culture, Maintenance and Chemical Induction}

Commercially available hMSCs with passage number 14 were utilized in this study (Steminent Biotherapeutics, Taipei, Taiwan). Cells were cultured in growth medium with the formation of Iscove's modified Dulbecco's Medium (IMDM, Invitrogen, Carlsbad, CA, USA), $10 \%$ fetal bovine serum (Sigma-Aldrich), $10 \mathrm{ng} / \mathrm{mL}$ basic fibroblast growth factor (Sigma-Aldrich, St. Louis, MO, USA), 100 units of penicillin, 1000 units of streptomycin, and $2 \mathrm{mM}$ L-glutamine (Sigma-Aldrich, St. Louis, MO, USA). The incubation condition was constantly set as $37^{\circ} \mathrm{C}, 5 \%$ carbon dioxide. Initially hMSCs were seeded at $30 \%$ confluent. After a week of expansion to reach the confluency of $80 \%$, the cells were detached by Trypsin-EDTA (Sigma-Aldrich, St. Louis, MO, USA). The cells were than harvested and introduced to 3D scaffolds with the density of $1.5 \times 105$ of cells per matrix after two passages of culture. Such timing was defined as day 0 .

For biochemical induction toward bone formation, the original medium was substituted with osteogenic regimen 1 day after hMSCs were administered on 3D matrices. The recipe was IMDM-based, supplemented with $0.1 \mu \mathrm{M}$ dexamethasone (Sigma-Aldrich, St. Louis, MO, USA), $10 \mathrm{mM}$ B-Glycerol Phosphate (Sigma-Aldrich, St. Louis, MO, USA), $1 \%$ penicillin-streptomycin- glutamine (Gibco BRL, Gaithersburg, MD, USA), and $0.2 \mathrm{mM}$ autologous serum-derived albumin (Sigma-Aldrich, St. Louis, MO, USA). The medium was provided for another 21 days and changed twice a week. The hMSCs were harvested on day 0 and 7, 14, 21 days post-induction for further assessments.

\subsection{RNA Isolation and Quantitative Real-Time Polymerase Chain Reaction}

Total RNA was extracted from cultured hMSCs with and without fluorescent bead injection on day 0 as well as 7, 14, 21 days post-induction by RNeasy Mini Kit (QIAGEN, Manchester, UK). Initially, lysis buffer, RLT solution with 1\% B-mercaptoethanol, and commercial kits were employed to isolate RNA means as usual. Yet due to excessive polyacrylamide debris of 3D scaffolds on the filtering membrane, the efficacy of RNA harvest was compromised and the purity was suboptimal. In response, trizol method was adapted. Chloroform was added after smashing the matrix with pipetman tips. polyacrylamide residues were aggregated at the interface of supernatant/organic layer after accelerated centrifugation. Subsequent double washing with alcohol further assured the immaculacy of RNA pallets. To reach adequate amount, the RNA was juiced from 1 slice of 2D hydrogel/24 pieces of 3D PA scaffolds per condition. $1 \mu \mathrm{g}$ of isolated RNA was reverse transcribed to complementary DNA using MMLV High Performance Reverse Transcriptase (EPICENTRE, Madison, WI, USA). Real-time polymerase chain reaction for quantification was accomplished with TaqMan Fast Universal PCR Master Mix. Two osteogeneic markers, Osterix and Runx2, were assessed.

\subsection{Statistical Analysis}

All exhibited statistics were presented as mean \pm standard error of mean and obtained in triplicate for statistical validation. three independent experiments. Quantitative results were analyzed by SPSS software (Version 19.0, IBM, Armonk, New York, USA) and com- 
pared by Student $t$-test. $p$ value $<0.05$ was considered significant and with $\left({ }^{*}\right)$ denotation, while ${ }^{* *}$ ) represented $p<0.01$.

\section{Conclusions}

The study pointed out the alteration of 3D matrix rigidity would orchestrate the mechanical parameters of cultured hMSCs. By our established platform for substrate manufacturing and VPTM protocol to assess Young's modulus, the $12 \mathrm{kPa}$ but not $1 \mathrm{kPa}$ matrix significantly facilitated the stiffening of hMSCs throughout the 21-day chemicallystimulated osteogenic course. Such characteristics may be further adapted for identification of osteogenic maturity in hMSCs, and may serve as a readout for the development of osteoinductive biomaterials.

Author Contributions: T.-W.K. conducted the cell culture, evaluations of viscoelasticities, and composed the article. A.C. established the apparatus of fluorescent bead injection and video particle tracking. K.-H.L. was prerequisite for the fabrication of 3D scaffolds. Y.-S.L. assisted on data analysis and interpretation. O.K.-S.L. supervised the whole program. All authors have read and agreed to the published version of the manuscript.

Funding: The authors declared financial support from the Ministry of Science and Technology, Taiwan (MOST 105-2815-C-010-001-B, MOST103-2314-B-010-053-MY3, MOST 106-2321-B-010-008, MOST 106-2911-I-010-502, MOST 106-3114-B-010-002) and Foundation for the Advancement of Outstanding Scholarship. This study was also supported by Aiming for the Top University Plan, a grant from Ministry of Education, Taiwan.

Data Availability Statement: The dataset supporting the conclusions of this article is included within the article.

Acknowledgments: The authors acknowledge financial support from the Ministry of Science and Technology (MOST 109-2926-I-010-501, MOST 107-2314-B-010-015-MY3, MOST 109-2926-I-010-502, MOST 109-2321-B-010-005, MOST 108-2923-B-010-002-MY3, MOST 109-2823-8-010-003-CV, MOST 1092622-B-010-006 and MOST 109-2321-B-010-006). This work is particularly supported by “Development and Construction Plan" of the School of Medicine, National Yang-Ming University, now known as National Yang Ming Chiao Tung University (107F-M01-0504) and Aiming for the Top University Plan, a grant from Ministry of Education.

Conflicts of Interest: The authors declare no conflict of interest. The funders had no role in the design of the study, in the collection, analyses, or interpretation of data, in the writing of the manuscript, or in the decision to publish the results.

\section{Appendix A}

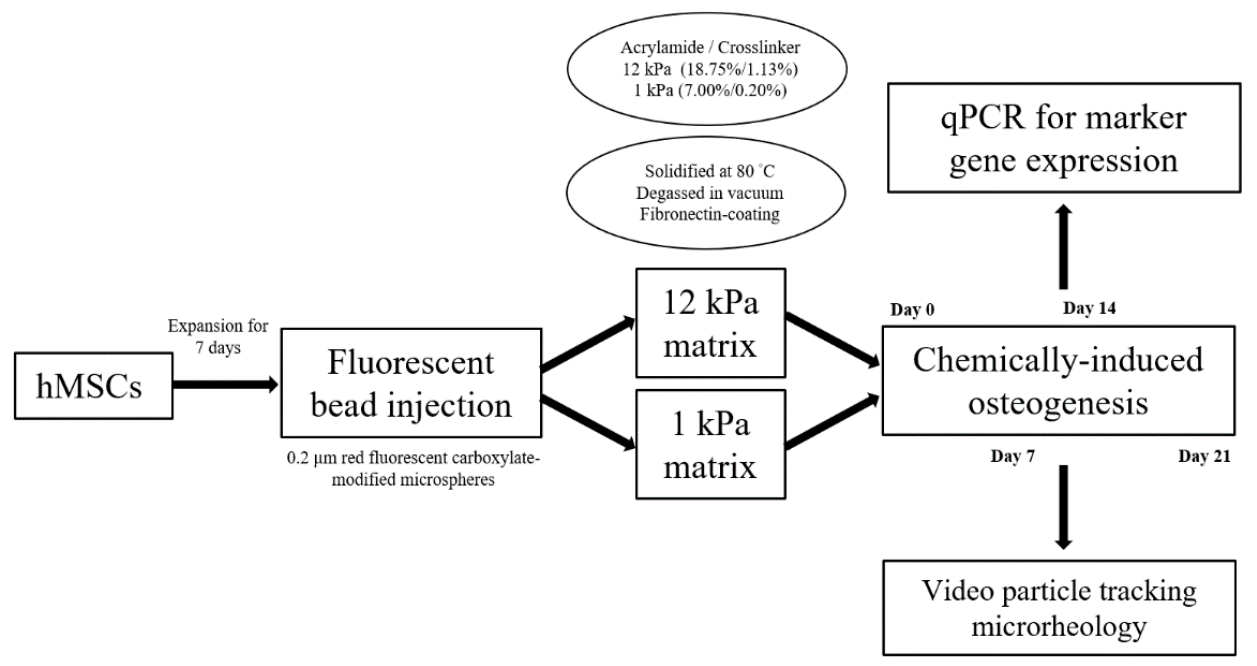

Figure A1. Study design. hMSC: human mesenchymal stem cell. qPCR: quantified polymerase chain reaction. 


\section{References}

1. Clynes, M.A.; Harvey, N.C.; Curtis, E.M.; Fuggle, N.R.; Dennison, E.M.; Cooper, C. The epidemiology of osteoporosis. Br. Med. Bull. 2020, 16, 105-117. [CrossRef] [PubMed]

2. Compston, J.E.; McClung, M.R.; Leslie, W.D. Osteoporosis. Lancet 2019, 393, 364-376. [CrossRef]

3. Cummings, S.R.; San Martin, J.; McClung, M.R.; Siris, E.S.; Eastell, R.; Reid, I.R.; Pierre Delmas, M.D.; Matt Austin, M.S.; Andrea Wang, M.A.; Stepan Kutilek, M.D.; et al. Denosumab for prevention of fractures in postmenopausal women with osteoporosis. N. Engl. J. Med. 2009, 361, 756-765. [CrossRef]

4. Engler, A.J.; Sen, S.; Sweeney, H.L.; Discher, D.E. Matrix elasticity directs stem cell lineage specification. Cell 2006, 126, 677-689. [CrossRef]

5. Khatiwala, C.B.; Kim, P.D.; Peyton, S.R.; Putnam, A.J. ECM compliance regulates osteogenesis by influencing MAPK signaling downstream of RhoA and ROCK. J. Bone Miner. Res. 2009, 24, 886-898. [CrossRef]

6. Wrighton, K.H. Mechanotransduction: YAP and TAZ feel the force. Nat. Rev. Mol. Cell Biol. 2011, 12, 404. [CrossRef]

7. Mullen, C.A.; Vaughan, T.J.; Billiar, K.L.; McNamara, L.M. The effect of substrate stiffness, thickness, and cross-linking density on osteogenic cell behavior. Biophys. J. 2015, 108, 1604-1612. [CrossRef]

8. Caiazzo, M.; Okawa, Y.; Ranga, A.; Piersigilli, A.; Tabata, Y.; Lutolf, M.P. Defined three-dimensional microenvironments boost induction of pluripotency. Nat. Mater. 2016, 15, 344-352. [CrossRef] [PubMed]

9. Baker, B.M.; Chen, C.S. Deconstructing the third dimension: How 3D culture microenvironments alter cellular cues. J. Cell Sci. 2012, 125, 3015-3024. [CrossRef]

10. Hsieh, W.T.; Liu, Y.S.; Lee, Y.H.; Rimando, M.G.; Lin, K.H.; Lee, O.K. Matrix dimensionality and stiffness cooperatively regulate osteogenesis of mesenchymal stromal cells. Acta Biomater. 2016, 32, 210-222. [CrossRef]

11. Kim, H.; Bae, C.; Kook, Y.M.; Koh, W.G.; Lee, K.; Park, M.H. Mesenchymal stem cell 3D encapsulation technologies for biomimetic microenvironment in tissue regeneration. Stem Cell Res. Ther. 2019, 10, 51. [CrossRef]

12. Yen, M.H.; Chen, Y.H.; Liu, Y.S.; Lee, O.K. Alteration of Young's modulus in mesenchymal stromal cells during osteogenesis measured by atomic force microscopy. Biochem. Biophys. Res. Commun. 2020, 526, 827-832. [CrossRef] [PubMed]

13. Mason, T.G. Estimating the viscoelastic moduli of complex fluids using the generalized Stokes-Einstein equation. Rheol. Acta 2000, 39, 371-378. [CrossRef]

14. Banks, J.M.; Mozdzen, L.C.; Harley, B.A.; Bailey, R.C. The combined effects of matrix stiffness and growth factor immobilization on the bioactivity and differentiation capabilities of adipose-derived stem cells. Biomaterials 2014, 35, 8951-8959. [CrossRef] [PubMed]

15. Keogh, M.B.; O'Brien, F.J.; Daly, J.S. Substrate stiffness and contractile behaviour modulate the functional maturation of osteoblasts on a collagen-GAG scaffold. Acta Biomater. 2010, 6, 4305-4313. [CrossRef]

16. Wirtz, D. Particle-tracking microrheology of living cells: Principles and applications. Annu. Rev. Biophys. 2009, 38, 301-326. [CrossRef]

17. McGlynn, J.A.; Wu, N.; Schultz, K.M. Multiple particle tracking microrheological characterization: Fundamentals, emerging techniques and applications. J. Appl. Phys. 2020, 127, 201101. [CrossRef]

18. Chen, Y.Q.; Chou, P.L.; Cheng, C.Y.; Chiang, C.C.; Wei, M.T.; Chuang, C.T.; Chen, Y.L.S.; Chiou, A. Microrheology of human synovial fluid of arthritis patients studied by diffusing wave spectroscopy. J. Biophoton. 2012, 5, 777-784. [CrossRef]

19. Chen, Y.Q.; Kuo, C.Y.; Wei, M.T.; Wu, K.; Su, P.T.; Huang, C.S.; Chiou, A.E. Intracellular viscoelasticity of HeLa cells during cell division studied by video particle-tracking microrheology. J. Biomed. Opt. 2014, 19, 011008. [CrossRef] [PubMed]

20. Lee, Y.H.; Huang, J.R.; Wang, Y.K.; Lin, K.H. Three-dimensional fibroblast morphology on compliant substrates of controlled negative curvature. Integr. Biol. Camb. 2013, 5, 1447-1455. [CrossRef] [PubMed]

21. Lo, Y.P.; Liu, Y.S.; Rimando, M.G.; Lin, K.H. Three-dimensional spherical spatial boundary conditions differentially regulate osteogenic differentiation of mesenchymal stromal cells. Sci. Rep. 2016, 6, 21253. [CrossRef] [PubMed]

22. Huebsch, N.; Arany, P.R.; Mao, A.S.; Shvartsman, D.; Ali, O.A.; Bencherif, S.A.; Rivera-Feliciano, J.; Mooney, D.J. Harnessing traction-mediated manipulation of the cell/matrix interface to control stem-cell fate. Nat. Mater. 2010, 9, 518-526. [CrossRef]

23. Haugh, M.G.; Vaughan, T.J.; Madl, C.M.; Raftery, R.M.; McNamara, L.M.; O’Brien, F.J.; Heilshorn, S.C. Investigating the interplay between substrate stiffness and ligand chemistry in directing mesenchymal stem cell differentiation within 3D macro-porous substrates. Biomaterials 2018, 171, 23-33. [CrossRef]

24. Li, Z.; Gong, Y.; Sun, S.; Du, Y.; Lü, D.; Liu, X.; Long, M. Differential regulation of stiffness, topography, and dimension of substrates in rat mesenchymal stem cells. Biomaterials 2013, 34, 7616-7625. [CrossRef]

25. Yu, L.; Wu, Y.; Liu, J.; Li, B.; Ma, B.; Li, Y.; Huang, Z.; He, J. 3D culture of bone marrow-derived mesenchymal stem cells could improve bone regeneration in 3D-printed porous Ti6Al4V scaffolds. Stem Cells Int. 2018, 2018, 2074021. [CrossRef]

26. Yao, B.; Wang, R.; Wang, Y.; Zhang, Y.; Hu, T.; Song, W.; Li, Z.; Huang, S.; Fu, X. Biochemical and structural cues of 3D-printed matrix synergistically direct MSC differentiation for functional sweat gland regeneration. Sci. Adv. 2020, 6, 1094. [CrossRef] [PubMed]

27. Egger, D.; Oliveira, A.C.; Mallinger, B.; Hemeda, H.; Charwat, V.; Kasper, C. From 3D to 3D: Isolation of mesenchymal stem/stromal cells into a three-dimensional human platelet lysate matrix. Stem Cell Res. Ther. 2019, 10, 248. [CrossRef] [PubMed] 
28. Wei, D.; Liu, A.; Sun, J.; Chen, S.; Wu, C.; Zhu, H.; Chen, Y.; Luo, H.; Fan, H. Mechanics-controlled dynamic cell niches guided osteogenic differentiation of stem cells via preserved cellular mechanical memory. ACS Appl. Mater. Interfaces 2020, 12, 260-274. [CrossRef]

29. Rabel, K.; Kohal, R.J.; Steinberg, T.; Tomakidi, P.; Rolauffs, B.; Adolfsson, E.; Palmero, P.; Fürderer, T.; Altmann, B. Controlling osteoblast morphology and proliferation via surface micro-topographies of implant biomaterials. Sci. Rep. 2020, 10, 12810 [CrossRef] [PubMed]

30. Becquart, P.; Cruel, M.; Hoc, T.; Sudre, L.; Pernelle, K.; Bizios, R.; Logeart-Avramoglou, D.; Petite, H.; Bensidhoum, M. Human mesenchymal stem cell responses to hydrostatic pressure and shear stress. Eur. Cell Mater. 2016, 31, 160-173. [CrossRef] [PubMed] 